\title{
Role of Sarcolemmal BKCa Channels in Stretch-Induced Extrasystoles in Isolated Chick Hearts
}

\author{
Gentaro Iribe, MD, PhD; Honghua Jin, PhD; Keiji Naruse, MD, PhD
}

\begin{abstract}
Background: It remains unclear whether sarcolemmal BKca channels in post-hatch chick ventricular myocytes contribute to stretch-induced extrasystoles (SIE), and whether they are stretch-activated BKca (SAKca) channels or a non-stretch-sensitive BKCa variant.

Methods and Results: To determine the role of sarcolemmal BKca channels in SIE and their stretch sensitivity, an isolated 2-week-old Langendorff-perfused chick heart and mathematical simulation were used. The ventricular wall was rapidly stretched by application of a volume change pulse. As the speed of the stretch increased, the probability of SIE also significantly increased, significantly shortening the delay between SIE and the initiation of the stretch. Application of $100 \mathrm{nmol} / \mathrm{L}$ of Grammostola spatulata mechanotoxin 4, a cation-selective stretch-activated channel (SAC) blocker, significantly decreased the probability of SIE. The application of Iberiotoxin, however, a BKca channel blocker, significantly increased the probability of SIE, suggesting that a $\mathrm{K}^{+}$efflux via a sarcolemmal BKCa channel reduces SIE by balancing out stretch-induced cation influx via SACs. The simulation using a cardiomyocyte model combined with a new stretch sensitivity model that considers viscoelastic intracellular force transmission showed that stretch sensitivity in BKca channels is required to reproduce the present wet experimental results.
\end{abstract}

Conclusions: Sarcolemmal BKca channels in post-hatch chick ventricular myocytes are SAKca channels, and they have a suppressive effect on SIE. (Circ J 2011; 75: 2552-2558)

Key Words: Arrhythmia; Modeling; Stretch-activated channels

$\mathbf{M}$ echanical stress affects various aspects of cardiovascular physiology. ${ }^{1-4}$ In particular, mechanically induced disturbance of heart rhythm is a phenomenon known as mechanoelectric feedback (MEF). ${ }^{2,5}$ For instance, a mechanical blunt impact to the chest can initiate life-threatening arrhythmia, known as commotio cordis. ${ }^{2} \mathrm{~A}$ precordial thump can also effectively terminate ventricular tachycardia and fibrillation. ${ }^{4}$

These mechanosensitive responses in heart rhythm are believed to be caused by activation of cation-selective stretchactivated channels (SACs). In a cross-circulated isolated canine heart, acute diastolic left ventricular (LV) wall stretch induced an extrasystolic beat that could be suppressed by $\mathrm{Gd}^{3+}$, a potent (but non-specific) SAC blocker. ${ }^{6}$ Furthermore, in a Langendorff-perfused rabbit heart, stretch-induced atrial fibrillation could be suppressed by Grammostola spatulata mechanotoxin 4 (GsMTx4), an SAC blocker. ${ }^{7}$ Although these findings strongly suggest the involvement of SAC in stretchinduced arrhythmia, it has also been reported that many stretch-sensitive channels are $\mathrm{K}^{+}$or anion selective, ${ }^{8-10}$ and their activation may stabilize membrane potential. Therefore, interactions between stretch-induced "destabilizer" and "stabilizer" channels need to be studied carefully to clarify the underlying mechanism of mechanically induced disturbance of heart rhythm.

We have previously reported the electrophysiological properties of stretch-activated $\mathrm{BK}$ Ca $\left(\mathrm{SAKC}_{\mathrm{C}}\right.$ ) channels cloned from cultured chick embryo ventricular myocytes. This type of channel is a voltage-sensitive $\mathrm{K}^{+}$channel, which is activated by intracellular $\mathrm{Ca}^{2+}\left(\left[\mathrm{Ca}^{2+}\right]_{i}\right)$ and membrane stretch. ${ }^{9} \mathrm{We}$ found that an SAKCa channel is a splicing variant of a $\mathrm{BKCa}$ channel, and includes a stress axis-regulated exon (STREX) sequence, which is responsible for its stretch sensitivity. ${ }^{11}$ Furthermore, we found that STREX variants of BKCa channels in chicks and humans share a short amino acid residue sequence (ERA) and show stretch sensitivity. STREX variants of these channels, however, in mice and rabbits that do not have the ERA sequence do not show stretch sensitivity. ${ }^{11}$ Although it is widely believed that sarcolemmal $\mathrm{BKCa}$ channels do not exist in mature ventricular myocytes, ${ }^{12}$ we reported the existence of sarcolemmal BKCa channels using direct micrographic evidence in post-hatch chick ventricular myocytes. ${ }^{13}$ It remains

Received May 9, 2011; revised manuscript received July 3, 2011; accepted July 11, 2011; released online September 14, 2011 Time for primary review: 17 days

Cardiovascular Physiology, Okayama University Graduate School of Medicine, Dentistry and Pharmaceutical Sciences, Okayama, Japan

The first two authors contributed equally to this work (G.I., H.J.).

Mailing address: Gentaro Iribe, MD, PhD, Cardiovascular Physiology, Okayama University Graduate School of Medicine, Dentistry and

Pharmaceutical Sciences, 2-5-1 Shikata-cho, Kita-ku, Okayama 700-8558, Japan. E-mail: iribe@md.okayama-u.ac.jp

ISSN-1346-9843 doi:10.1253/circj.CJ-11-0486

All rights are reserved to the Japanese Circulation Society. For permissions, please e-mail: cj@j-circ.or.jp 


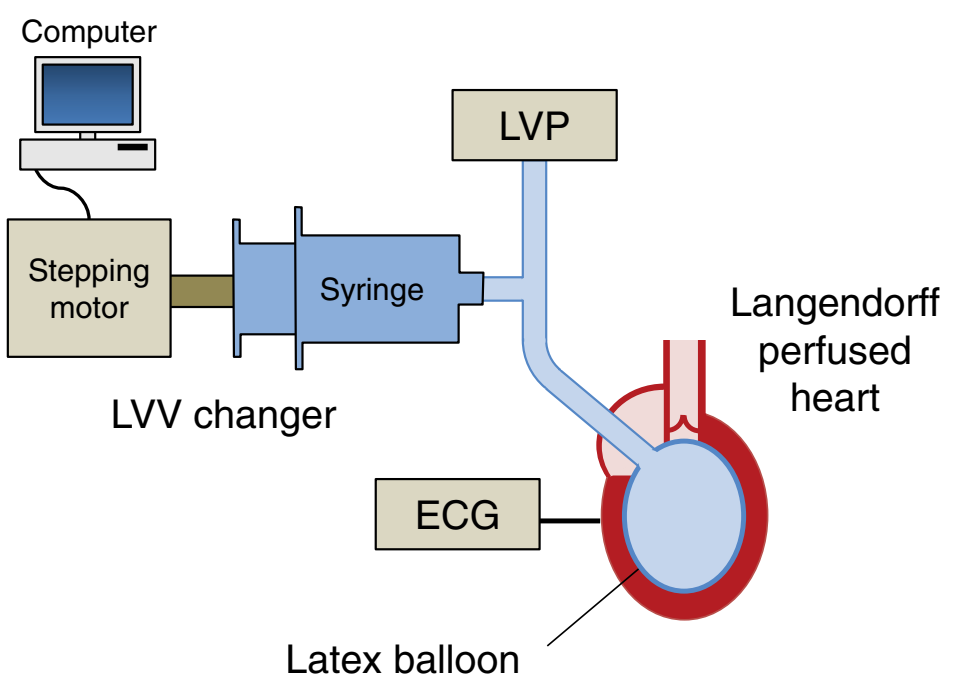

Figure 1. Schematic diagram of the experimental preparation. Left ventricular (LV) volume (LVV) was controlled by changing the volume of a latex balloon inserted into the LV. LV pressure (LVP) and electrocardiogram (ECG) were monitored.
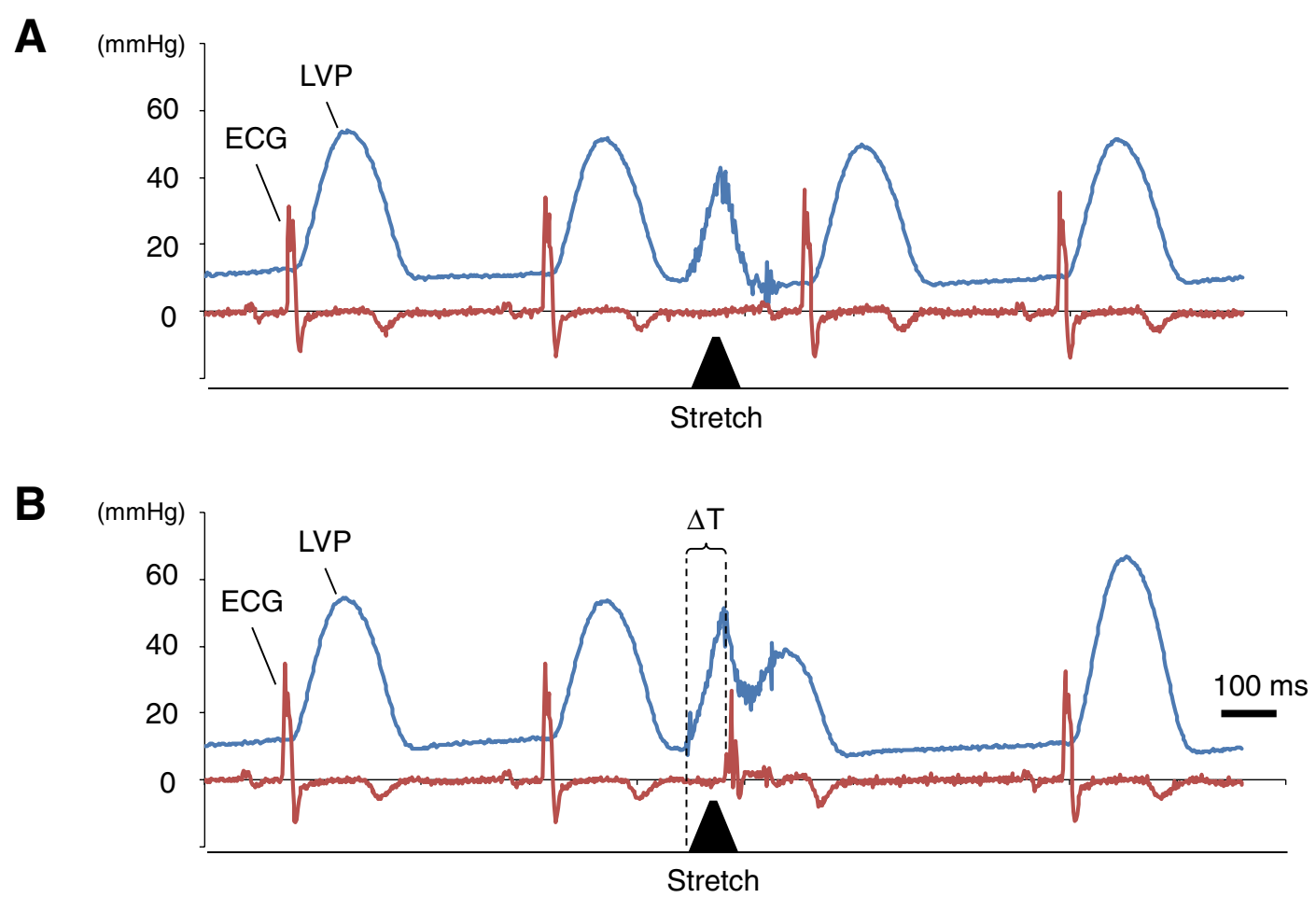

Figure 2. Representative electrocardiogram (ECG) and left ventricular pressure (LVP) traces with diastolic ventricular wall stretch. (A) When a stretch did not initiate stretch-induced extrasystoles (SIE), no ectopic QRS complex corresponding to the stretch was present on ECG. (B) When a stretch initiated SIE, ectopic QRS complex sets appeared on ECG after a delay $(\Delta T)$ from the beginning of the stretch signal.

unclear, however, whether these channels are SAKCa or a non-stretch-sensitive BKCa variant.

In the present study, we investigated the involvement of SACs and sarcolemmal BKCa channels in stretch-induced extrasystoles (SIE) using isolated Langendorff-perfused chick hearts with a computer-controlled LV stretch system. We also explored the possibilities of stretch sensitivity in sarcolemmal
$\mathrm{BK}_{\mathrm{Ca}}$ channels using experimental results in combination with a mathematical simulation.

\section{Methods}

All experiments were performed according to the Guiding Principles for the Care and Use of Animals approved by the 

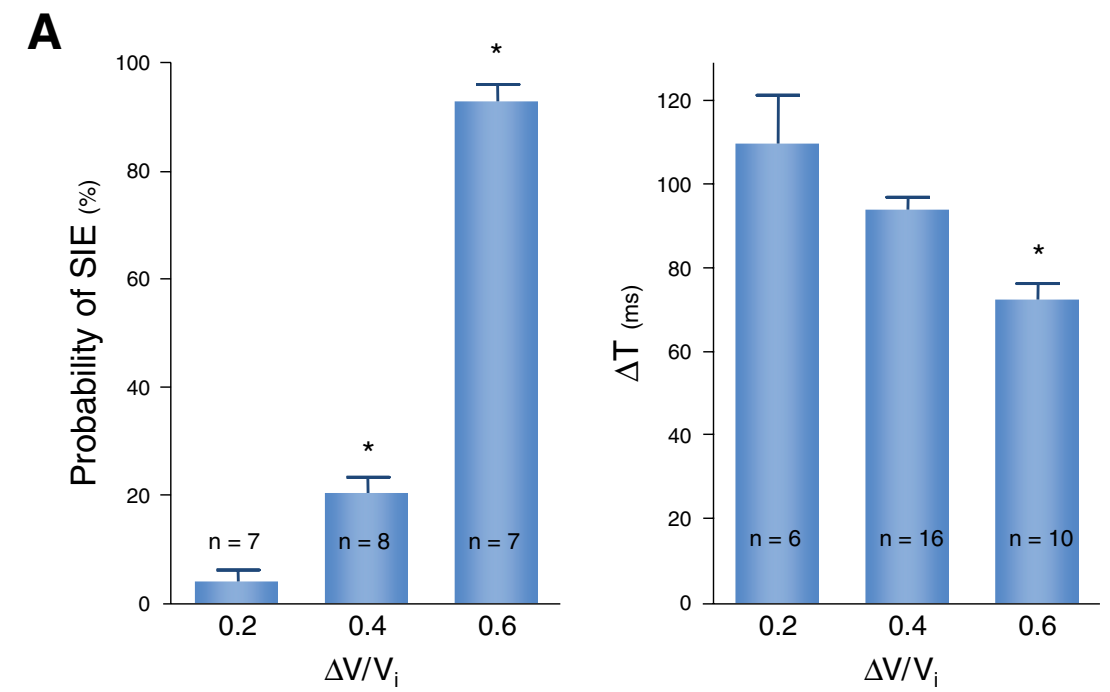

B
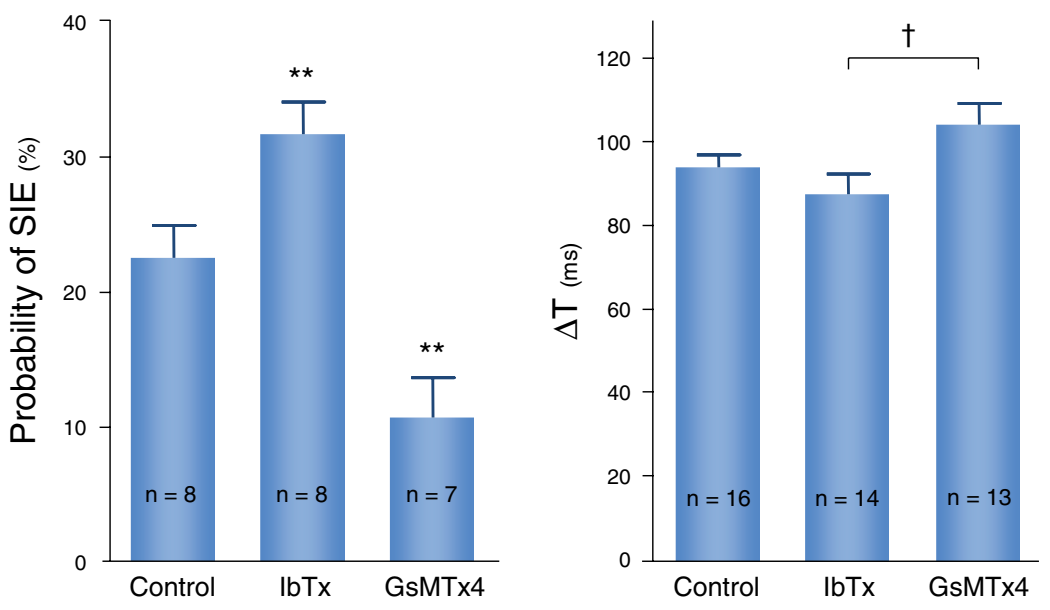

Figure 3. Probability of stretch-induced extrasystoles (SIE) and $\Delta \mathrm{T}$. (A) As $\Delta \mathrm{V} / \mathrm{Vi}$ increased, the probability of SIE increased, and $\Delta T$ shortened significantly. (B) Effects of Iberiotoxin (IbTx), a BKca channel blocker, and Grammostola spatulata mechanotoxin 4 (GsMTx4), a stretch-activated channel (SAC) blocker, on the probability of SIE and $\Delta T$ at a medium stretch $(\Delta \mathrm{V} / \mathrm{Vi}=0.4)$. IbTx increased the probability of SIE significantly, and showed a tendency to decrease $\Delta T$. GsMTX4 decreased the probability of SIE significantly, and showed a tendency to increase $\Delta \mathrm{T}$. Vi, initial left ventricular volume. ${ }^{\star} \mathrm{P}<0.05$ vs. $\Delta \mathrm{V} / \mathrm{Vi}=0.2 .{ }^{* *} \mathrm{P}<$ 0.05 vs. control; ${ }^{\mathrm{t} P}<0.05$ vs. IbTx.

Council of the Physiological Society of Japan. The study protocol was approved by the Animal Subjects Committee of Okayama University Graduate School of Medicine, Dentistry, and Pharmaceutical Sciences.

\section{Heart Preparation}

Two-week-old chicks (body weight 90-120 g) were heavily anesthetized with ether. After median sternotomy, the heart was rapidly excised from the chest and washed in cold normal Tyrode (NT) solution $(\mathrm{NaCl}, 136 \mathrm{mmol} / \mathrm{L} ; \mathrm{KCl}, 5.4 \mathrm{mmol} / \mathrm{L}$; $\mathrm{MgCl}_{2}, 1 \mathrm{mmol} / \mathrm{L} ; \mathrm{NaH}_{2} \mathrm{PO}_{4}, 0.3 \mathrm{mmol} / \mathrm{L} ; \mathrm{CaCl}_{2}, 1.8 \mathrm{mmol} / \mathrm{L}$; glucose, $10 \mathrm{mmol} / \mathrm{L}$; HEPES, $5 \mathrm{mmol} / \mathrm{L}$; $\mathrm{pH} 7.4)$. The pericardium was then removed. The aorta was cannulated and 2 major branches from the trunk of the aorta were ligated. The heart was then perfused with NT solution at a constant flow of $12 \mathrm{ml} / \mathrm{min}$ using a flow roller pump system. The left atrium appendage was opened and a thin latex balloon with an unstressed volume of $150 \mu \mathrm{l}$ was fitted into the LV chamber. The balloon was connected to our custom-made LV volume (LVV) changer and a pressure transducer; the circuit was primed with water. The LVV changer consisted of a $0.5-\mathrm{ml}$ syringe connected to a rapid microstepping motor (Figure 1). The stepper was controlled by custom software written in LabView (National Instruments, Austin, TX, USA). Electrocardiogram (ECG) and LV pressure
(LVP) were continuously monitored. The data were digitized and acquired online at $500 \mathrm{~Hz}$ using custom LabView software and a fast analogue-to-digital/digital-to-analogue interface (NI DAQCard-6062E; National Instruments). The temperature of the heart was maintained at $37^{\circ} \mathrm{C}$, and the perfusate was oxygenated throughout the experiment.

\section{Experiment Protocol}

Initial LVV (Vi) was adjusted such that the diastolic LVP was $10 \mathrm{mmHg}(35-45 \mu \mathrm{l})$. After obtaining a stable and regular beating state under sinus rhythm, a transient LV wall stretch pulse (40-ms upstroke, 10-ms plateau, 40-ms downstroke) was applied by the LVV changer during the diastolic phase (Figure 2A). The upstroke of LVP was used as a trigger to identify the start of the cardiac cycle, and an approximately 200-ms delay was inserted before the pulse. The delay time was adjusted to assure that the pulse was applied after LVP returned to the diastolic pressure. We fixed the upstroke duration of the LV wall stretch pulse; therefore, the rate of stretch depended on the amount of LVV change $(\Delta \mathrm{V})$. The ratio of $\Delta \mathrm{V}$ against $\mathrm{Vi}$ $(\Delta \mathrm{V} / \mathrm{Vi})$ was set at $0.2,0.4$, and 0.6 to apply a small (slow), medium, and large (fast) LV wall stretch, respectively. We repeated the stretch 20 times at each $\Delta \mathrm{V} / \mathrm{Vi}$, and calculated the percentage of SIE occurrence as the probabilities of SIE. 


\section{Mathematical Modeling}

Mathematical simulations are based on a ventricular myocyte model (Iribe-Kohl-Noble 200814), modified by the addition of a $\mathrm{BK}_{\mathrm{Ca}}$ channel $^{15}$ and $\mathrm{SAC}^{16}$ with stretch sensitivity that considers the viscoelastic property of the cytoplasm for force transmission. We modified the length-dependent stretch sensitivity equations from those of the Oxsoft model ${ }^{16}$ by adding a simple Maxwell-type local viscoelastic cytoplasmic unit that consists of a viscous damper and an elastic spring connected in series. We used a cytoplasm elastic modulus $(E)$ of $10 \mathrm{kPa}$, obtained from chick cardiomyocytes,${ }^{17}$ and a viscosity $(\eta)$ of $2 \mathrm{kPa} \cdot \mathrm{s}$, obtained from the adherent cell surface. ${ }^{18}$ The resultant stress relaxation curve (decay time constant, $0.2 \mathrm{~s}$ ) is reasonably comparable to the experimentally obtained creep response on the local cell surface. ${ }^{18}$

$$
\begin{aligned}
\frac{d \varepsilon 1}{d t} & =S_{\text {stretch }}-\frac{E \varepsilon 1}{\eta} \\
\frac{d \varepsilon 2}{d t} & =\frac{E \varepsilon 1}{\eta}
\end{aligned}
$$

Where $\varepsilon_{1}$ and $\varepsilon_{2}$ are strains of the spring and damper of the viscoelastic component, respectively, and $S_{\text {stretch }}$ is the speed of the stretch. We used yielded tension $(E \varepsilon 1)$ and sarcomere length $(S L)$ as a source function for the speed-dependent activation factor ( $\left.f_{\text {stretch_speed }}\right)$ and length-dependent activation factor $\left(f_{\text {stretch_length }}\right)$, respectively. To our knowledge, there are no previous studies that characterized electrophysiological properties of speed dependency. Therefore, we determined the parameters for calculating $f_{\text {stretch_speed }}$ to reproduce the experimental results of speed dependency (Figure 3A) in a qualitative manner within the rate of stretch we used. The parameters for calculating $f_{\text {stretch_length }}$ are the same as in the original Oxsoft model ${ }^{16}$ except for reduced gain (0.01), used to reproduce the results in our previous single cell study,,$^{13}$ and slow force response (SFR) to stretch. ${ }^{19}$ The final stretchdependent activation factor $\left(f_{\text {stretch }}\right)$ was calculated as follows:

$$
\begin{aligned}
& f_{\text {stretch }}=f_{\text {stretch_speed }}+f_{\text {stretch_length }} \\
& f_{\text {stretch_speed }}=\frac{1}{1+e^{-6(E \varepsilon 1-1.4)}} \\
& f_{\text {stretch_length }}=\frac{0.01}{1+e^{-5(S L-2.0)}}
\end{aligned}
$$

The cell model was coded in CellML format, and run using COR (Cellular Open Resource: http://cor.physiol.ox.ac.uk/). All cell model equations are given in Data S1.

\section{Data Analysis}

All data are presented as mean \pm SEM. Statistical assessment was performed using 1-way analysis of variance followed by Bonferroni's multiple comparison test. $\mathrm{P}<0.05$ was considered to be statistically significant.

\section{Results}

There were no significant differences in heart rates and QT intervals among the $3 \Delta \mathrm{V} / \mathrm{Vi}$ groups (Table).

Figure 2 shows representative ECG and LVP traces with diastolic ventricular wall stretch $(\Delta \mathrm{V} / \mathrm{Vi}=0.6)$. SIE occurs in a stochastic manner. In cases in which SIE was not induced, the wall stretch resulted in a diastolic LVP increase because of LVV change, and no ectopic QRS complexes corresponding to the stretch were observed on ECG (Figure 2A). When SIE was induced, however, QRS complex and T wave sets appeared for which the pattern differed from regular beats.

\begin{tabular}{|cccc}
\hline \multicolumn{4}{l}{ Table. HR and QT Interval vs. $\Delta \mathbf{V} / \mathbf{V i}$} \\
\multicolumn{1}{c}{$\Delta \mathbf{V} / \mathbf{V i}$} & $\mathbf{0 . 2}(\mathbf{n}=\mathbf{6})$ & $\mathbf{0 . 4}(\mathbf{n}=\mathbf{1 6})$ & $\mathbf{0 . 6}(\mathbf{n}=\mathbf{9})$ \\
HR (beats/min) & $127.0 \pm 10.6$ & $121.5 \pm 3.5$ & $120.9 \pm 2.0$ \\
QT interval (ms) & $236.3 \pm 9.9$ & $238.1 \pm 5.7$ & $245.3 \pm 8.743$ \\
\hline
\end{tabular}

$\mathrm{HR}$, heart rate; Vi, initial left ventricular volume.

Ectopic beats emerged without the preceding $\mathrm{P}$ wave, and usually with a compensatory pause (longer beat interval after an extrasystolic event). Furthermore, a delay between SIE and initiation of stretch $(\Delta \mathrm{T})$ was observed. The stretch induced a triangular diastolic LVP increase because of LVV change. This was followed by another increase in LVP from an ectopic LV contraction when SIE was initiated (Figure 2B).

Figure $3 \mathrm{~A}$ shows the probability of SIE and $\Delta \mathrm{T}$ of the SIE for the $3 \Delta \mathrm{V} / \mathrm{Vi}$ groups. The left panel shows the averages of probabilities of SIE of all hearts (" $n$ " indicates the number of hearts analyzed). Then the SIE beats for which ECG traces are clear enough to analyze $\Delta \mathrm{T}$ were selected, and the data were averaged and shown in the right panel (" $n$ " indicates the number of beats analyzed). As $\Delta \mathrm{V} / \mathrm{Vi}$ increased, the probability of SIE increased and $\Delta \mathrm{T}$ was shortened significantly. A shorter $\Delta \mathrm{T}$ indicates that the stretch initiated SIE rapidly; in other words, it easily initiates SIE and therefore, the results in both panels are consistent.

To elucidate the involvement of SAC and sarcolemmal BKCa channels in SIE, we applied either $100 \mathrm{nmol} / \mathrm{L}$ of GsMTx4, an SAC blocker, or $100 \mathrm{nmol} / \mathrm{L}$ of Iberiotoxin (IbTx), a BKCa channel blocker, during repeats of the same SIE experiments at a medium stretch $(\Delta \mathrm{V} / \mathrm{Vi}=0.4)$. As shown in Figure $3 \mathrm{~B}$, GsMTx 4 decreased the probability of SIE significantly and showed a tendency to increase $\Delta \mathrm{T}(\mathrm{P}=0.06)$. IbTx, however, increased the probability of SIE significantly and showed a tendency to decrease $\Delta \mathrm{T}(\mathrm{P}=0.07)$. Thus, suggesting that a polarizing effect of $\mathrm{K}^{+}$efflux via the sarcolemmal $\mathrm{BK}$ ca channel reduces SIE by balancing the depolarizing effects of the stretch-induced cation influx via SACs.

The SAKCa channel was initially cloned from a chick embryo heart. Recently, we also found sarcolemmal BKCa channel currents in more mature ventricular single myocytes isolated from post-hatch chick heart, but it remains unclear whether these channels are $\mathrm{SAK}$ Ca channels or other non-stretch-sensitive $\mathrm{BK}_{\mathrm{Ca}}$ variants. To examine $\mathrm{BK}_{\mathrm{Ca}}$ channel behavior in SIE with and without stretch sensitivity, we simulated the present SIE protocol using a mathematical cardiomyocyte model with $\mathrm{SAK}_{\mathrm{Ca}}$ (with stretch sensitivity) or $\mathrm{BKCa}$ (without stretch sensitivity) channels.

To simulate SIE, we applied a triangular pulse (50-ms upstroke, 50-ms downstroke: total $100 \mathrm{~ms}$ ) to the SL change during the diastolic phase of $2-\mathrm{Hz}$ stimulation (start to stretch $250 \mathrm{~ms}$ after the preceding stimulation). Figure 4 shows simulated SIE in the model with SAKCa (panel A) and BKCa (panel B). We tested 3 different rates of stretch $(3.0,4.0$ and $6.0 \mu \mathrm{m} / \mathrm{s}$ in SL). The faster stretch increased $f_{\text {stretch }}$ (stretch-dependent activation factor) more quickly. The larger the $f_{\text {stretch, the larger }}$ the SAC current (ISAC) we could observe until the membrane potential reached the excitation threshold. Once the membrane is excited, Isac becomes outward current due to positive membrane potential, so that inward peak current resulted at around $2 \mathrm{nA}$ regardless of $f_{\text {stretch }}$ value. In both models, the inward IsAC depolarized the membrane. With the slowest stretch $(3.0 \mu \mathrm{m} / \mathrm{s}$ in SL), the depolarization did not reach the threshold to initiate an action potential, whereas with the faster stretches (4.0 and $6.0 \mu \mathrm{m} / \mathrm{s}$ in SL) it evoked ectopic action potentials. 
A

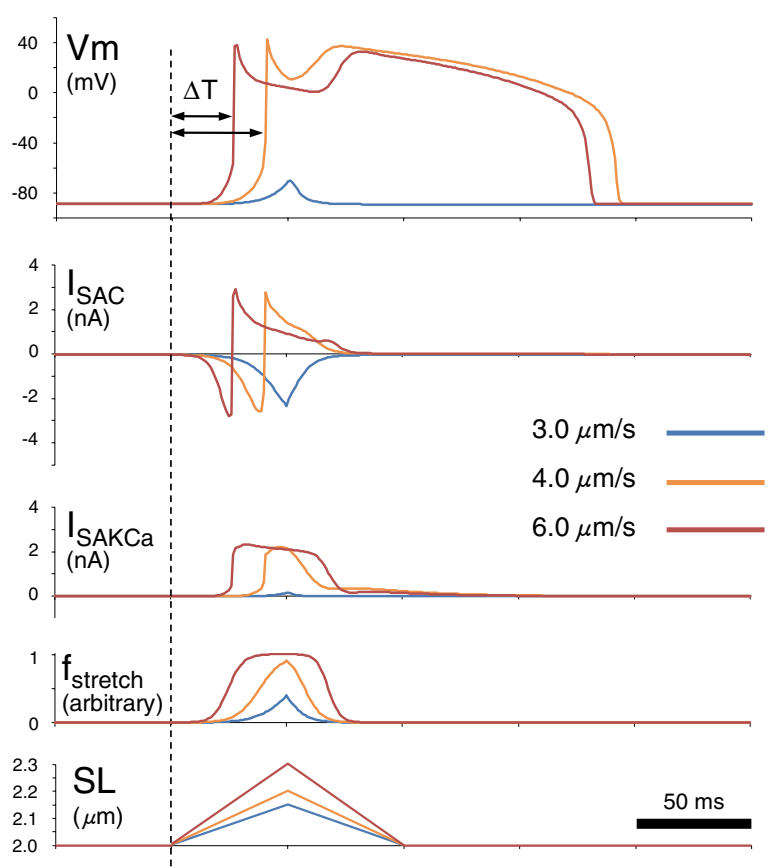

\section{B}

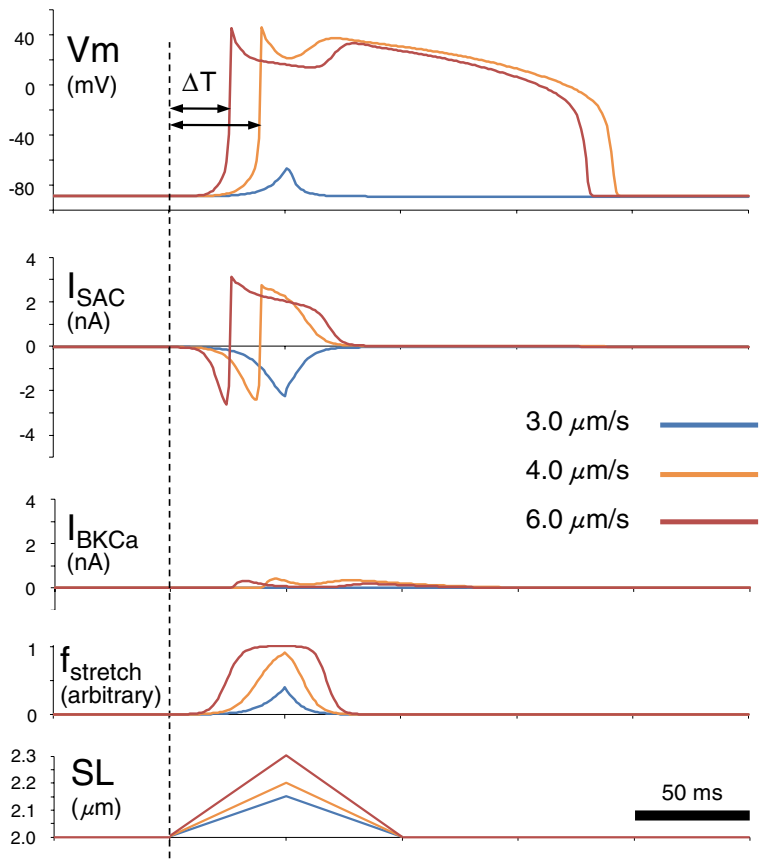

Figure 4. Mathematical model of stretch-induced extrasystoles (SIE) at 3 different stretch speeds with (A) stretch-activated BKCa (SAKca; with stretch sensitivity) and (B) BKCa (without stretch sensitivity). The faster stretch resulted in a greater $f_{\text {stretch }}$ and inward IsAC, producing quicker excitation (shorter $\Delta \mathrm{T}$ ). This tendency did not differ between SAKca and BKCa. IsAC, stretch-activated channel

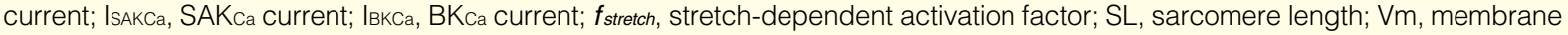
potential.

The fastest stretch $(6.0 \mu \mathrm{m} / \mathrm{s}$ in SL) had a shorter $\Delta \mathrm{T}$ and a steeper inward slope for IsAC compared to that produced by medium stretch $(4.0 \mu \mathrm{m} / \mathrm{s}$ in SL). Both models reproduced the present wet experimental results shown in Figure $\mathbf{3 A}$.

The present wet experimental results show that an IbTx-sensitive current contributes to a decrease in the probability of SIE (Figure 3B). To examine whether SAKCa current (IsAKCa) and/or $\mathrm{BK}$ са current (IвKCa) have an effect of suppressing SIE, these currents were increased by changing their conductance (GKCa in Eq. A23; Data S1) in the model with the medium stretch speed $(4.0 \mu \mathrm{m} / \mathrm{s}$ in SL). As shown in Figure $\mathbf{5 A}$, increasing IsAKCa by a 5-fold increase in GKCa abolished SIE. In contrast, as shown in Figure 5B, increasing IвKCa by a 20 -fold increase in GKCa did not inhibit SIE, even though its peak value is comparable to the increased SAKCa current in Figure 5A. These results can be explained as follows. Without stretch sensitivity, outward $\mathrm{K}^{+}$current that counterbalances inward IsAC is absent until the cell is excited and $\left[\mathrm{Ca}^{2+}\right] \mathrm{i}$ has to be increased to activate $\mathrm{BK}_{\mathrm{Ca}}$ channels (period between $a$ and $b$ in Figure 5B). Furthermore, when the Iвкса appears, the cell is already excited and remains excited even when the Iвкса is increased. In contrast, with stretch sensitivity, SAKCa channels can be activated by stretch without an increase in $\left[\mathrm{Ca}^{2+}\right]_{\mathrm{i}},{ }^{23}$ and the outward IsAKCa balances the inward IsAC. Therefore, IsAKCa during stretch before excitation (period between $a$ and $b$ in Figure 5A) is important to prevent the membrane potential from reaching the excitation threshold. The simulation results suggest that the IbTx-sensitive current must be stretch sensitive in order to explain the results seen in Figure 3B.

\section{Discussion}

The present wet and dry experiments clarified 2 findings. The first is that the IbTx-sensitive current reduces the probability of SIE, suggesting that it counterbalances stretch-induced inward IsAc. The second is that the counterbalancing outward current is suspected to respond quickly to the stretch before excitation, otherwise it cannot prevent a stretch-evoked action potential. Our previous single channel studies have also demonstrated that activation of SAKCa channels by stretch occurs immediately after the stretch is applied. ${ }^{9}$ Therefore, we concluded that the IbTx-sensitive channels in chick heart are SAKCa channels.

Our previous study in post-hatch chick ventricular isolated single myocytes, however, demonstrated that the IbTx-sensitive outward current did not increase as a primary immediate response to a slow axial stretch using carbon fiber technique. Instead, the outward current gradually increased as the stretch was sustained over several minutes. ${ }^{13}$ These results suggest that sarcolemmal $\mathrm{BK}_{\mathrm{Ca}}$ channels were not primarily activated by stretch, but rather were secondarily activated by a stretchinduced increase in $\left[\mathrm{Ca}^{2+}\right]$. If the sarcolemmal BKca channels in chick heart are SAKCa channels, why were they not immediately activated by slow axial stretch? Probably, they could respond to a rapid stretch, but not to a slow stretch. Therefore, in the present modeling study, we assumed that the mechanosensitive channels are mainly activated in a speed-dependent manner and that the length-dependent factor is very small. It is well known that a mechanical stimulus transmitted via 
A

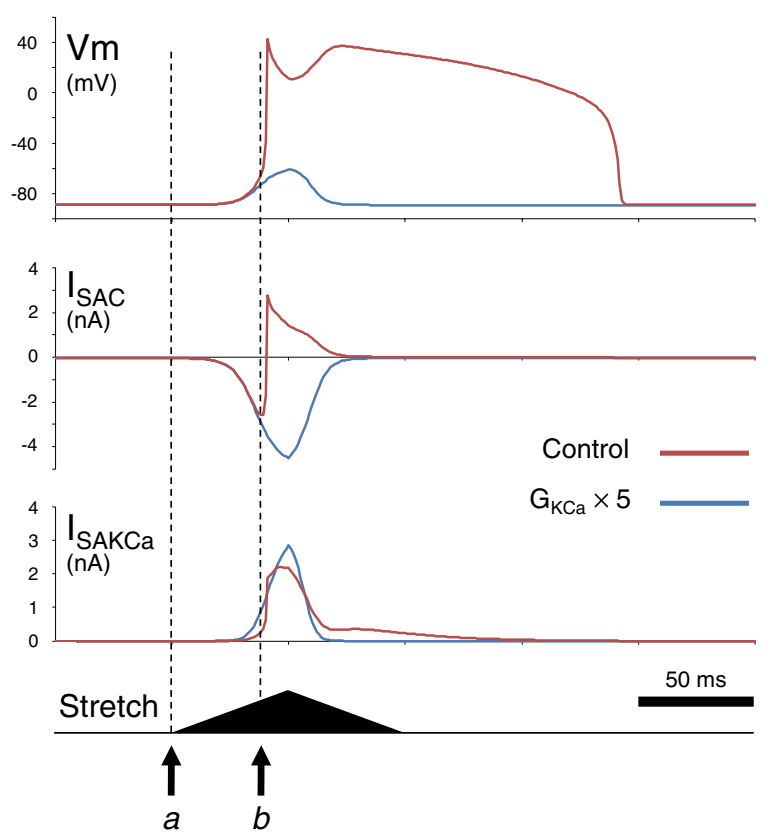

B

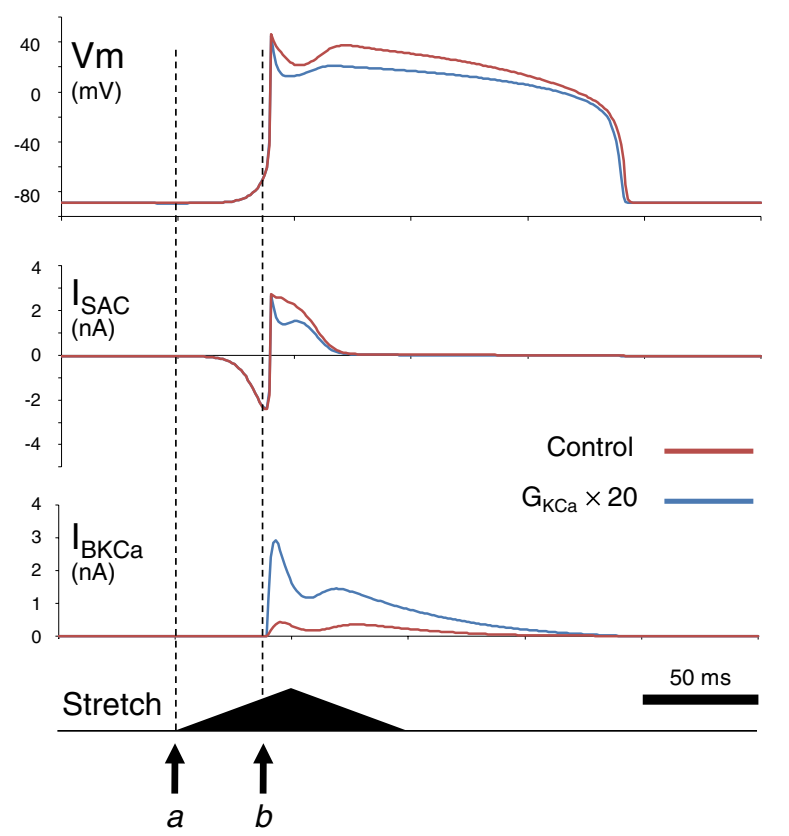

Figure 5. Mathematical model of stretch-induced extrasystoles (SIE) with increased (A) stretch-activated BKca (SAKca; with stretch sensitivity) and (B) BKca (without stretch sensitivity) currents. The speed of the stretch was fixed at $4.0 \mu \mathrm{m} / \mathrm{s}$. (A) A 5-fold increase in SAKca conductance increased outward ISAKCa that counterbalances inward IsAC during stretch before excitation (period between $a$ and $b$ ), thereby abolishing SIE. (B) Even though a 20 -fold increase in BKCa conductance produced a greater peak outward lBkCa than IsAKCa in (A), it did not affect outward current during stretch before excitation, thereby not affecting SIE occurrence. $a$, beginning of the stretch; $b$, beginning of excitation in control state; GKCa, conductance of SAKca or BKca channels; IsAc, stretch-activated channel current; IsAKCa, SAKca current; IвKCa, BKca current; Vm, membrane potential.

the cytoskeleton activates mechanosensitive channels. ${ }^{24,25}$ Therefore, it is reasonable that the viscoelastic property of the cytoskeleton (stress fibers) ${ }^{26}$ causes stretch speed-dependent activation of mechanosensitive channels.,25 This hypothesis explains the discrepancy between the present results and previous results from a slow stretch single cell study. ${ }^{13}$

To obtain direct evidence of speed-dependent activation, it is necessary to study SIE in single cell preparations using an electrophysiological approach. Although recent developments in stretching isolated single cardiomyocytes using carbon fiber techniques allow mechanical load to be considered in cellular physiology studies, ${ }^{14,27,28}$ it is still not easy to study SIE in single cell preparations because of difficulties in applying a quick stretch during patch-clamp experiments. Although whole heart preparations are advantageous when applying a quick mechanical load, they are not suitable for studying subcellular mechanisms, including ion channel properties. Therefore, mathematical modeling can be used to connect both sets of findings to predict the underlying mechanisms in SIE.

Stretching cardiac muscle shows an immediate increase in force (Frank-Starling mechanism) followed by a delayed increase in developed force that takes place over several minutes. The slow secondary response to stretch is called SFR. ${ }^{19}$ The SFR is associated with an increase in $\mathrm{Ca}^{2+}$ handling due to a stretch-induced increase in $\mathrm{Na}^{+}$influx, followed by extrusion of $\mathrm{Na}^{+}$accompanied by $\mathrm{Ca}^{2+}$ influx via the $\mathrm{Na}^{+} / \mathrm{Ca}^{2+}$ exchanger. ${ }^{29}$ Length-dependent activation of SACs cannot explain SIE and SFR simultaneously by itself because, as shown in Figure 4,
SIE usually requires a substantial current to increase the membrane potential to the threshold level. If such a large inward current continues when the stretch is sustained under lengthdependent activation, myocardial excitation-contraction coupling may collapse rapidly. Furthermore, the required cation (probably $\mathrm{Na}^{+}$) influx for SFR is small and such a small current cannot induce SIE. In contrast, the taking into account of speed-dependent activation can explain SIE and SFR using the same model. We successfully reproduced SFR with the same model as in Figure 4A, but a slow stretch from $\mathrm{SL}=2.0$ to $2.3 \mu \mathrm{m}(0.4 \mu \mathrm{m} / \mathrm{s}$ for $0.75 \mathrm{~s})$ was applied. Although the total amount of stretch $(0.3 \mu \mathrm{m}$ in SL) was the same as that observed with the fastest stretch $(6.0 \mu \mathrm{m} / \mathrm{s}$ for $0.05 \mathrm{~s}$; Figure $4 \mathrm{~A})$, we did not observe any ectopic excitation, rather a gradual increase in twitch force due to a small SAC-mediated $\mathrm{Na}^{+}$ influx followed by a secondary increase in $\left[\mathrm{Ca}^{2+}\right]$ i through $\mathrm{Na}^{+} / \mathrm{Ca}^{2+}$ exchanger activity (data not shown).

Nevertheless, many previous findings have indicated that length-dependent activation contributes to SIE. For instance, in a canine whole heart preparation with an LV wall stretch experiment similar to that used in the present study, the probability of SIE increased when the LV wall stretch pulse duration was increased, suggesting that wall distension itself contributed to initiation of SIE. In a single rat myocyte, the stretchinduced increase in membrane potential was dependent on the amount of stretch; ${ }^{25}$ with the same amount of stretch, the change in membrane potential was faster with a faster stretch, but the actual amount of change remained the same at dif- 
ferent speeds. ${ }^{25}$ These behaviors look length dependent, possibly because of the unknown time-dependent activating and/or inactivating factor that we did not take into account in the present stretch-sensitivity model. Although single SAKCa channel current traces in the previous study indicated that their response to stretch was quick enough, the detailed time-dependent property was not fully studied. ${ }^{9}$ Therefore, the present stretch sensitivity model is limited in its ability to explain all known findings in MEF, but it at least does suggest the involvement of speed in the present results.

Another potential limitation in the present model is that the model took into account neither SL change nor force change due to the contraction itself, for simplicity. Although effects of contraction are not crucial in terms of the initiation of SIE by a diastolic stretch, still its overall effects would be important for other MEF phenomena that the present model does not explain.

In conclusion, we studied the involvement of sarcolemmal BKCa channels in SIE in isolated chick heart using a combination of a computer-controlled LV stretch system and mathematical simulation. We found that the IbTx-sensitive $\mathrm{BK}_{\mathrm{Ca}}$ current reduced the probability of SIE. The present modeling study suggests that the sarcolemmal $\mathrm{BK}_{\mathrm{Ca}}$ channels in chick heart need to be stretch-sensitive SAKca channels to inhibit SIE.

\section{Acknowledgment}

This work was supported by the Japan Society for the Promotion of Science (JSPS KAKENHI: 19200037, 20300159, 20034038 and 22136001).

\section{References}

1. Ando J, Yamamoto K. Vascular mechanobiology: Endothelial cell responses to fluid shear stress. Circ J 2009; 73: 1983-1992.

2. Kohl P, Hunter P, Noble D. Stretch-induced changes in heart rate and rhythm: Clinical observations, experiments and mathematical models. Prog Biophys Mol Biol 1999; 71: 91-138.

3. Koyama T, Ono K, Watanabe H, Ohba T, Murakami M, Iino K, et al. Molecular and electrical remodeling of L- and T-type $\mathrm{Ca}^{2+}$ channels in rat right atrium with monocrotaline-induced pulmonary hypertension. Circ J 2009; 73: 256-263.

4. Pellis T, Kohl P. Extracorporeal cardiac mechanical stimulation: Precordial thump and precordial percussion. Br Med Bull 2010; 93: $161-177$.

5. Lab MJ, Taggart P, Sachs F. Mechano-electric feedback. Cardiovasc Res 1996; 32: 1-2.

6. Hansen DE, Borganelli M, Stacy GP Jr, Taylor LK. Dose-dependent inhibition of stretch-induced arrhythmias by gadolinium in isolated canine ventricles: Evidence for a unique mode of antiarrhythmic action. Circ Res 1991; 69: 820-831.

7. Bode F, Sachs F, Franz MR. Tarantula peptide inhibits atrial fibrillation. Nature 2001; 409: 35-36.

8. Hagiwara N, Masuda H, Shoda M, Irisawa H. Stretch-activated anion currents of rabbit cardiac myocytes. J Physiol 1992; 456: 285-302.

9. Kawakubo T, Naruse K, Matsubara T, Hotta N, Sokabe M. Characterization of a newly found stretch-activated $\mathrm{KCa}_{\mathrm{C}}$, ATP channel in cultured chick ventricular myocytes. Am J Physiol 1999; 276: H1827-H1838.

10. Ruknudin A, Sachs F, Bustamante JO. Stretch-activated ion channels in tissue-cultured chick heart. Am J Physiol 1993; 264: H960-H972.

11. Naruse K, Tang QY, Sokabe M. Stress-Axis Regulated Exon (STREX) in the C terminus of BKCa channels is responsible for the stretch sensitivity. Biochem Biophys Res Commun 2009; 385: 634639.
12. Sargent CA, Grover GJ, Antonaccio MJ, McCullough JR. The cardioprotective, vasorelaxant and electrophysiological profile of the large conductance calcium-activated potassium channel opener NS-004. J Pharmacol Exp Ther 1993; 266: 1422-1429.

13. Iribe G, Jin H, Kaihara K, Naruse K. Effects of axial stretch on sarcolemmal BKCa channels in post-hatch chick ventricular myocytes. Exp Physiol 2010; 95: 699-711.

14. Iribe $\mathrm{G}, \mathrm{Kohl} \mathrm{P}$. Axial stretch enhances sarcoplasmic reticulum $\mathrm{Ca}^{2+}$ leak and cellular $\mathrm{Ca}^{2+}$ reuptake in guinea pig ventricular myocytes: Experiments and models. Prog Biophys Mol Biol 2008; 97: 298311.

15. Moczydlowski E, Latorre R. Gating kinetics of $\mathrm{Ca}^{2+}$-activated $\mathrm{K}^{+}$ channels from rat muscle incorporated into planar lipid bilayers: Evidence for two voltage-dependent $\mathrm{Ca}^{2+}$ binding reactions. J Gen Physiol 1983; 82: 511-542.

16. Kohl P, Day K, Noble D. Cellular mechanisms of cardiac mechanoelectric feedback in a mathematical model. Can J Cardiol 1998; 14: $111-119$.

17. Hofmann UG, Rotsch C, Parak WJ, Radmacher M. Investigating the cytoskeleton of chicken cardiocytes with the atomic force microscope. J Struct Biol 1997; 119: 84-91.

18. Bausch AR, Ziemann F, Boulbitch AA, Jacobson K, Sackmann E. Local measurements of viscoelastic parameters of adherent cell surfaces by magnetic bead microrheometry. Biophys J 1998; 75: 2038-2049.

19. Allen DG, Kurihara S. The effects of muscle length on intracellular calcium transients in mammalian cardiac muscle. J Physiol 1982; 327: 79-94.

20. Noble D, Noble SJ, Bett GC, Earm YE, Ho WK, So IK. The role of sodium-calcium exchange during the cardiac action potential. Ann NY Acad Sci 1991; 639: 334-353.

21. Noble D, Varghese A, Kohl P, Noble P. Improved guinea-pig ventricular cell model incorporating a diadic space, IKr and IKs, and length- and tension-dependent processes. Can J Cardiol 1998; 14: $123-134$.

22. Rice JJ, Winslow RL, Hunter WC. Comparison of putative cooperative mechanisms in cardiac muscle: Length dependence and dynamic responses. Am J Physiol 1999; 276: H1734-H1754.

23. Zhao H, Sokabe M. Tuning the mechanosensitivity of a BK channel by changing the linker length. Cell Res 2008; 18: 871-878.

24. Hayakawa K, Tatsumi H, Sokabe M. Actin stress fibers transmit and focus force to activate mechanosensitive channels. J Cell Sci 2008; 121: $496-503$.

25. Nishimura S, Kawai Y, Nakajima T, Hosoya Y, Fujita H, Katoh M, et al. Membrane potential of rat ventricular myocytes responds to axial stretch in phase, amplitude and speed-dependent manners. Cardiovasc Res 2006; 72: 403-411.

26. Kumar S, Maxwell IZ, Heisterkamp A, Polte TR, Lele TP, Salanga M, et al. Viscoelastic retraction of single living stress fibers and its impact on cell shape, cytoskeletal organization, and extracellular matrix mechanics. Biophys J 2006; 90: 3762-3773.

27. Iribe G, Helmes M, Kohl P. Force-length relations in isolated intact cardiomyocytes subjected to dynamic changes in mechanical load. Am J Physiol Heart Circ Physiol 2007; 292: H1487-H1497.

28. Iribe G, Ward CW, Camelliti P, Bollensdorff C, Mason F, Burton RA, et al. Axial stretch of rat single ventricular cardiomyocytes causes an acute and transient increase in $\mathrm{Ca}^{2+}$ spark rate. Circ Res 2009; 104: 787-795.

29. Calaghan S, White E. Activation of $\mathrm{Na}^{+}-\mathrm{H}^{+}$exchange and stretchactivated channels underlies the slow inotropic response to stretch in myocytes and muscle from the rat heart. $J$ Physiol 2004; 559: $205-214$.

\section{Supplemental Files}

Supplemental File 1

Data S1. All Mathematical Modeling Equations List

Data S2. All Model Parameters List

Please find supplemental file(s);

http://dx.doi.org/10.1253/circj.CJ-11-0486 